\title{
Dynamic Birefringence Behavior of Semicrystalline Polymers. I. Principles and Experimental Procedure of the $\pi$-Sector Technique ${ }^{\dagger}$
}

\author{
Thein Kyu, Naoshi Yasuda, Muneo Tabushi, ${ }^{*}$ Shunji Nomura, ${ }^{* *}$ \\ and Hiromichi KaWAI*** \\ Department of Polymer Chemistry, Faculty of Engineering, \\ Kyoto University, Kyoto, Japan.
}

(Received August, 9, 1974)

\begin{abstract}
An apparatus for the simultaneous observation of dynamic birefringence and dynamic modulus has been developed to pursue the responses of internal structures of semicrystalline polymers, i.e., the orientation processes of some structural units, in response to mechanical stimulation. This apparatus is similar in principle to the dynamic $\mathrm{X}$-ray diffraction device developed in the same laboratory, but the construction is partially modified. The $\pi$-sector technique is used in the measurement and its principles and application to dynamic birefringence are presented. The major problems lowering the precision of the measurement, which arose mainly from the fluctuations of the signals and drift of the amplifiers, have been overcome by amplifying the net amplitude of the dynamic signals and averaging the signals, thus achieving high accuracy. A good correlation between the mechanical and birefringence dispersions for a low-density polyethylene is observed and these results are discussed briefly.

KEY WORDS: Dynamic Birefringence / Semicrystalline Polymers / $\pi$-Sector Technique / Low Density Polyethylene / Complex Dynamic Strain Optical Coefficient /
\end{abstract}

In recent years, several speculations on the nature of mechanical loss processes of semicrystalline polymers have been proposed from mechanical ${ }^{1-14}$ and dielectric ${ }^{15,16}$ studies. The mechanical loss peaks are thought to be associated with the onset of motions of polymer molecules within the crystal lattice, boundary surfaces, or lattice defects of polymer crystals. However, a measurement of mechanical properties merely observes the macroscopic mechanical response as a result of a combined contribution of the responses of internal structural units (crystalline, amorphous, and other intermediate

† A part of the M.S. thesis of Thein Kyu, presented to the Department of Polymer Chemistry, Faculty of Engineering, Kyoto University, on February 26, 1974.

* Present address: Kawasaki Mill, Nippon Unicar Co., Ltd., Kawasaki, Japan.

** On leave to the Divison of Macromolecular Science, Case Western Reserve University, Cleveland, Ohio 44106, U.S.A.

*** To whom correspondence should be addressed. phases) to the stimulation of the external bulk strain, therefore mechanical studies alone are inadequate and cannot account for the behavior of the internal structures during relaxation.

To achieve direct information from the internal structures, the so-called rheo-optical techniques have been developed by several authors: ${ }^{17-23}$ optical quantities having direct correlation with definite structural units and their orientation are studied simultaneously with the mechanical measurements. The rheo-optical studies could offer significant information about the behavior of the internal structures allowing a comparison of the relaxation behavior of such optical quantities with the simultaneously observed mecanical relaxation behavior. It has been demonstrated in relaxation $^{31-34}$ and vibrational birefringence ${ }^{24-26,29,30}$ that the strain optical coefficient is time dependent; this is attributed to the change of crystal orientation accompaning the amorphous orientation. The time-dependence of the orientation of polymer crystals has been also confirmed by relaxation $^{35,36}$ and vibrational ${ }^{26,29} \mathrm{X}$-ray diffrac- 
tion studies, in which the orientation behavior of the principal crystallographic axes of polyethylene crystallites are explained in terms of reorientation processes within the deformed spherulite. The time dependence of the spherulitic deformations of polyethylene has also been demonstrated by dynamic light scattering studies. ${ }^{37-39}$

Indeed, the purpose of the rheo-optical studies is to achieve direct evidence for understanding the mechanical loss mechanisms in terms of the response of definite structural units. However, measurements of dynamic rheo-optical properties are not necessarily easy, since the optical responses of the internal structural units during sinusoidal oscillation of the bulk strain are usually very small, especially when one keeps the mechanical responses within the limit of linear behavior. Therefore, it may be rather difficult to achieve a high precision by ordinary methods, such as the Lissajous' figure method..$^{23,24}$ Hence, it is worthwhile to develop a device of high accuracy to facilitate reliable measurements for rheo-optical studies.

Among the optical quantities, birefringence follows very sensitively the response of the structural units and is one of the most useful quantities for pursuing the orientation behavior of internal structures. The application of birefringence to dynamic mechanical measurements is, however, limited by the difficulty that the transmitted light intensity variation is rather small as compared to the static baseline intensity. This difficulty becomes severe especially in poorly transparent specimens. But, such difficulty has been overcome by the development of the socalled $\pi$-sector technique, which was previously utilized in dynamic X-ray diffraction studies. ${ }^{27,28}$

The $\pi$-sector technique, in principle, could offer better accuracy, but other problems are still involved in the measurement. The major problems which lower the precision of the measurement are the fluctuation of output signals and the drift of the amplifiers. The problem of fluctuation may be eliminated, since the fluctuation can be averaged out by carrying on the experiment over many cycles using the $\pi$ sector technique. But the problem of drift cannot be eliminated by the $\pi$-sector technique unless the amplitude of the dynamic signal is comparatively large. For this purpose, amplificasion of the dynamic signal is performed after diminishing the static base line with the aid of the operational amplifier, in order that only the net amplitude of the signal is amplified.

In this paper, the principles of the $\pi$-sector technique and its application to dynamic birefringence measurements are introduced. The modified portion of the deformation device and the detecting system are also briefly outlined. Experiments on a low-density polyethylene are reported over a frequency range of 0.008 to 4.3 $\mathrm{Hz}$ at temperatures from 30 to $80^{\circ} \mathrm{C}$. A good correlation between mechanical and birefringent dispersions is observed, in agreement with relaxational and vibrational birefringence studies of the materials previously reported. ${ }^{24,25,31,32}$

\section{Phenomenological Theory}

When a polymer film is subjected to a sinusoidal deformation, the resulting stress $\sigma$ and birefringence $\Delta n$ may vary sinusoidally by the phase difference angles $\delta$ and $\alpha$ respectively with respect to the dynamic bulk strain $\varepsilon$. These can be generally described as follows:

$$
\begin{gathered}
\varepsilon(t)=\Delta \varepsilon \exp i \omega t \\
\sigma(t)=\Delta \sigma \exp i(\omega t+\delta) \\
\Delta n(t)=\Delta(\Delta n) \exp i(\omega t+\alpha)
\end{gathered}
$$

where $\Delta \varepsilon, \Delta \sigma$, and $\Delta(\Delta n)$ are the amplitudes of the respective dynamic quantities and $\omega$ represents the angular frequency in radians. The phase difference angles with a positive sign connote that the stress and birefringence lead the prescribed strain; conversely, the negative sign represents a lag in phase.

The complex dynamic tensile modulus function $E^{*}(i \omega)$, which represents the mechanical response of the polymer to the stimulation of the external bulk strain, may be expressed as follows:

$$
E^{*}(i \omega)=(\sigma / \varepsilon)=E^{\prime}(\omega)+i E^{\prime \prime}(\omega)
$$

where the prime and double prime represent the real and imaginary parts of the complex dynamic modulus. Furthermore,

$$
\tan \delta=E^{\prime \prime} / E^{\prime} \text {. }
$$

Likewise, when the optical response of the polymer is observed, a similar formulation may be employed for the strain optical coefficient, as follows: 


$$
K^{*}(i \omega)=(\Delta n / \varepsilon)=K^{\prime}(\omega)+i K^{\prime \prime}(\omega)
$$

where $K^{*}$ is the complex dynamic strain optical coefficient, and the prime and double prime of $K$ represent the real and imaginary parts of the complex dynamic strain optical coefficient. Similarly,

$$
\tan \alpha=K^{\prime \prime} / K^{\prime}
$$

As the strain, stress, and birefringence are interrelated, the complex dynamic stress optical coefficient $M^{*}(i \omega)$ may be defined by the following similar formula:

$$
\begin{gathered}
M^{*}(i \omega)=(\Delta n / \sigma)=M^{\prime}(\omega)-i M^{\prime \prime}(\omega) \\
\tan \beta=\tan (\delta-\alpha)=M^{\prime \prime} / M^{\prime}
\end{gathered}
$$

where $\beta$ represents the phase difference angle between the stress and the birefringence.

\section{Principles of the $\pi$-Sector Technique and Its Ap- plication to Dynamic Birefringence ${ }^{30}$}

The block diagram for the simultaneous measurement of dynamic birefringence and dynamic modulus functions by the $\pi$-sector technique is shown in Figure 1. In principle, the $\pi$-sector technique is a method in which the produced signals, such as strain, stress, and birefringence, are converted to electric signals which may then be accumulated for relative

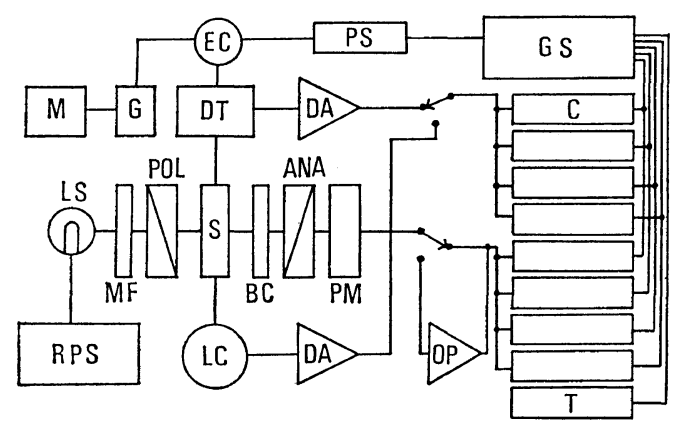

Figure 1. Block diagram for the dynamic birefringence apparatus using the $\pi$-sector technique: (LS) light source; (MF) monochromatic filter; (POL) polarizer; (S) specimen; (BC) Berek's compensator; (ANA) analyzer; (PM) photomultiplier; (RPS) regulated power supply; (LC) load cell; (DT) differential transformer; (DA) differential transformer amplifier; (OP) operational amplifier; (EC) eccentric cam; (M) motor; (G) reduction gear; (PS) photoelectric switch; (GS) gating system; (C) digital volt meter; and (T) timer. phase intervals of $\pi$-radians by digital volt meters. The real parts of the $\varepsilon, \sigma$, and $\Delta n$ signals may be given as follows:

$$
\begin{gathered}
\varepsilon(t)=\varepsilon_{0}+\Delta \varepsilon \cos \omega t \\
\sigma(t)=\sigma_{0}+\Delta \sigma \cos (\omega t+\delta) \\
\Delta n(t)=\Delta n_{0}+\Delta(\Delta n) \cos (\omega t+\alpha) .
\end{gathered}
$$

The symbols with the subscript 0 denote the base signals corresponding to static levels. The above signals may be transformed to voltage signals under the condition that there exist linear relationships between the strain, stress, birefringence, and the corresponding voltage signals $E_{\varepsilon}, E_{\sigma}$, and $E_{\Delta n}$ :

$$
\begin{gathered}
E_{\varepsilon}(t)=E_{\varepsilon_{0}}+\Delta E_{\varepsilon} \cos \omega t \\
E_{\sigma}(t)=E_{\sigma_{0}}+\Delta E_{\sigma} \cos (\omega t+\delta) \\
E_{\Delta n}(t)=E_{\Delta n_{0}}+\Delta E_{\Delta n} \cos (\omega t+\alpha)
\end{gathered}
$$

provided that

$$
\varepsilon(t)=m_{1} E_{\varepsilon}(t)+k_{1}
$$

where $m_{1}$ represents the gradient of the strain and its corresponding voltage signal and $k_{1}$ is a proportionality constant. Likewise, a similar condition is essential in the cases of the stress and the birefringence:

$$
\begin{gathered}
\sigma(t)=m_{2} E_{\sigma}+k_{2} \\
\Delta n(t)=m_{3} E_{\Delta n}+k_{3} .
\end{gathered}
$$

Taking the converted signal of the strain as a reference signal, four photoelectric switches are arranged so as to produce pulse signals for gating at particular phase angles of the dynamic strain, i.e., four angular positions of $0, \pi / 2, \pi$, and $3 \pi / 2$ radians, respectively, as shown in Figure 5. But in practice, it is quite difficult to set them up exactly at the above mentioned angular positions, so they are therefore adjusted with a deviation angle $\xi$. Then the output signals in the phase intervals of $\pi$ radians are accumulated by digital volt-meters. The accumulated counts in volts may be evaluated by the following equations. In the case of the strain signal accumulated in the phase interval of $0+\xi$ to $\pi+\xi$,

$$
N_{1}^{\varepsilon}=\frac{n}{\omega} \int_{\xi}^{\pi+\xi}\left[E_{\varepsilon_{0}}+\Delta E_{\varepsilon} \cos \omega t\right] \mathrm{d} \omega t
$$




$$
=\frac{n}{\omega}\left[\pi E_{\varepsilon_{0}}-2 \Delta E_{\varepsilon} \sin \xi\right]
$$

where $n$ and $\omega$ are the numbers of accumulating cycles and angular frequency. Similarly, the accumulated counts $\left(N_{2}{ }^{8}, N_{3}{ }^{8}\right.$, and $\left.N_{4}{ }^{8}\right)$ in the intervals of $\pi / 2+\xi$ to $3 \pi / 2+\xi, \pi+\xi$ to $2 \pi+\xi$, and $3 \pi / 2+\xi$ to $5 \pi / 2+\xi$ may be obtained. Then,

$$
\tan \xi=\left(N_{3}{ }^{\varepsilon}-N_{1}^{\varepsilon}\right) /\left(N_{4}^{\varepsilon}-N_{2}{ }^{\varepsilon}\right) .
$$

A similar derivation may be carried out in the cases of stress and birefringence to give

$$
\begin{aligned}
N_{1}{ }^{\sigma} & =\frac{n}{\omega} \int_{\xi}^{\pi+\xi}\left[E_{\sigma_{0}}+\Delta E_{\sigma} \cos (\omega t+\delta)\right] \mathrm{d} \omega t \\
& =\frac{n}{\omega}\left[\pi E_{\sigma_{0}}-2 \Delta E_{\sigma} \sin (\xi+\delta)\right] .
\end{aligned}
$$

$N_{2}{ }^{\sigma}, N_{3}{ }^{\sigma}$, and $N_{4}{ }^{\sigma}$ may be similarly derived. Hence,

$$
\tan (\xi+\delta)=\left(N_{3}{ }^{\sigma}-N_{1}{ }^{\sigma}\right) /\left(N_{4}{ }^{\sigma}-N_{2}{ }^{\sigma}\right) .
$$

From eq 18 and $20, \tan \delta$ can easily be obtained by the following equation:

$$
\tan \delta=[\tan (\xi+\delta)-\tan \xi] /[1+\tan \xi \tan (\xi+\delta)] \text {. }
$$

Furthermore, the absolute modulus $\left|E^{*}\right|$ may be calculated as follows:

$$
\begin{aligned}
\left|E^{*}\right| & =\frac{m_{2} \Delta E_{\sigma}}{m_{1} \Delta E_{\varepsilon}} \\
& =\left(m_{2} / m_{1}\right)\left[\frac{\left(N_{3}^{\sigma}-N_{1}{ }^{\sigma}\right)^{2}+\left(N_{4}{ }^{\sigma}-N_{2}{ }^{\sigma}\right)^{2}}{\left(N_{3}{ }^{\varepsilon}-N_{1}{ }^{\varepsilon}\right)^{2}+\left(N_{4}{ }^{\varepsilon}-N_{2}{ }^{2}\right)^{2}}\right]^{1 / 2} .
\end{aligned}
$$

In the case of birefringence, a similar formulation may be performed to evaluate $\tan \alpha$ and $\left|K^{*}\right|$ :

$$
\tan (\xi+\alpha)=\left(N_{3}{ }^{\Delta n}-N_{1}^{\Delta n}\right) /\left(N_{4}{ }^{\Delta n}-N_{2}{ }^{\Delta n}\right)
$$

$\tan \alpha=[\tan (\xi+\alpha)-\tan \xi] /[1+\tan \xi \tan (\xi+\alpha)]$

and

$$
\begin{aligned}
\left|K^{*}\right| & =\frac{m_{3} \Delta E_{\Delta n}}{m_{1} \Delta E_{\Delta n}} \\
& =\left(m_{3} / m_{1}\right)\left[\frac{\left(N_{3}{ }^{\Delta n}-N_{1}^{\Delta n}\right)^{2}+\left(N_{4}{ }^{\Delta n}-N_{2}{ }^{\Delta n}\right)^{2}}{\left(N_{3}{ }^{6}-N_{1}{ }^{\varepsilon}\right)^{2}+\left(N_{4}{ }^{6}-N_{2}{ }^{6}\right)^{2}}\right]^{1 / 2}
\end{aligned}
$$

where $m_{2}$ and $m_{3}$ are the gradients of the stress and birefringence and their respective voltage signals. As the complex dynamic stress optical coefficient $M^{*}$ and $\tan \beta$ are interrelated with those of the strain and birefringence, they may be easily derived to give the following relations:

$$
\begin{gathered}
\left|M^{*}\right|=\left|K^{*}\right| /\left|E^{*}\right| \\
\tan \beta=\tan (\delta-\alpha) \\
=(\tan \delta-\tan \alpha) /(1+\tan \delta \tan \alpha) .
\end{gathered}
$$

\section{Optical System}

The optical system comprises of two fundamental sections: the light source and the detecting system. A Nikon monofilament bulb $(30 \mathrm{~W}$, $6 \mathrm{~V}$ ) is used as the light source since it is essential to carry out the experiment over a long period. In this regard, the Nikon monofilament bulb is suitable for use as a stable light source, but in view of the present technology, a laser light source may be more appropriate. The incident beam is monochromatized by a monochromatic filter of wavelength $546 \mathrm{~m} \mu$. Then the specimen in ribbon shape is set in the clamps of the deformation device, as shown in Figure 1, so that its surface normal is parallel to the incident beam but its stretching direction inclines with $45^{\circ}$ against the polarization directions of the cross polarizers (polarizer and analyser). A compensator is placed between the specimen and the analyzer, as illustrated in Figure 1. The light intensity transmitted through the cross polarizers is determined by means of a photomultiplier, and may be represented as follows:

$$
I_{\mathrm{obs}}=I_{0}(\psi) \sin ^{2}(\psi+\phi) / 2
$$

where $\phi$ and $\phi$ are the phase retardation angles due to the compensator and the specimen.

In practice, however, the observed transmitted light intensity in terms of volts generated by the photomultiplier varies in such curved fashions, as shown later in Figure 6, with respect to the displacement of the compensator, which is related to the retardation angles $(\psi+\phi)$, and the degree of stretching for a given specimen, that in some portions the curves are practically linear. These linear portions of the curves are used for the determination of $I_{\mathrm{obs}}$. Over such a portion, one can assume that the observed intensity $I_{\text {obs }}$ varies linearly with the retardation angles $(\psi+\phi)$. But, if the compenensator (Berek's type) is fixed at the linear portion, $\phi$ becames constant, there- 
fore $I_{\mathrm{obs}}$ may vary directly with the retardation angle of the specimen, e.g., around $(\psi+\phi)=\pi / 2$,

$$
I_{\mathrm{obs}}=(1 / 2) I_{0}(\psi)[1-(\pi / 2)+(\psi+\phi)] .
$$

The birefringence of the specimen is defined by the relative retardation in $\mathrm{cm}$ thickness:

$$
\phi=\phi_{1}-\phi_{2}=(2 \pi d / \lambda) \Delta n
$$

where $\lambda$ is the wavelength of the light within the specimen, while $d$ represents the thickness of the specimen. In the case of dynamic birefringence, the birefringence $\Delta n$ varies sinusoidally as described in eq 3 ; but considering the real part only, and substituting it into eq 12,

$$
\phi=(2 \pi d / \lambda)\left[\Delta n_{0}+\Delta(\Delta n) \cos (\omega t+\alpha)\right] .
$$

Again, substituting eq 31 into eq 29 ,

$$
\begin{aligned}
I_{\mathrm{obs}}= & \frac{1}{2}\left[1-\left(\frac{\pi}{2}\right)+\psi+\frac{2 \pi d}{\lambda} \Delta n_{0}\right] I_{0} \\
& +\frac{\pi d}{\lambda} I_{0} \Delta(\Delta n) \cos (\omega t+\alpha) .
\end{aligned}
$$

The relative intensity alternation observed by the photomultiplier during the sinusoidal deformation of the specimen may be converted to a voltage signal; then eq 32 can be rewritten as

$$
E_{\Delta n}=E_{\Delta n_{0}}+\Delta E_{\Delta n} \cos (\omega t+\alpha)
$$

where $E_{\Delta n_{0}}$ and $\Delta E_{\Delta n}$ are the base voltage and the amplitude of the electrically converted signal of the birefringence, which is exactly the same as eq $12^{\prime}$.

\section{Device and Control Systems}

The specimen deformation device used in this study is similar to the device previously reported in the dynamic X-ray diffraction study; ${ }^{28}$ it was

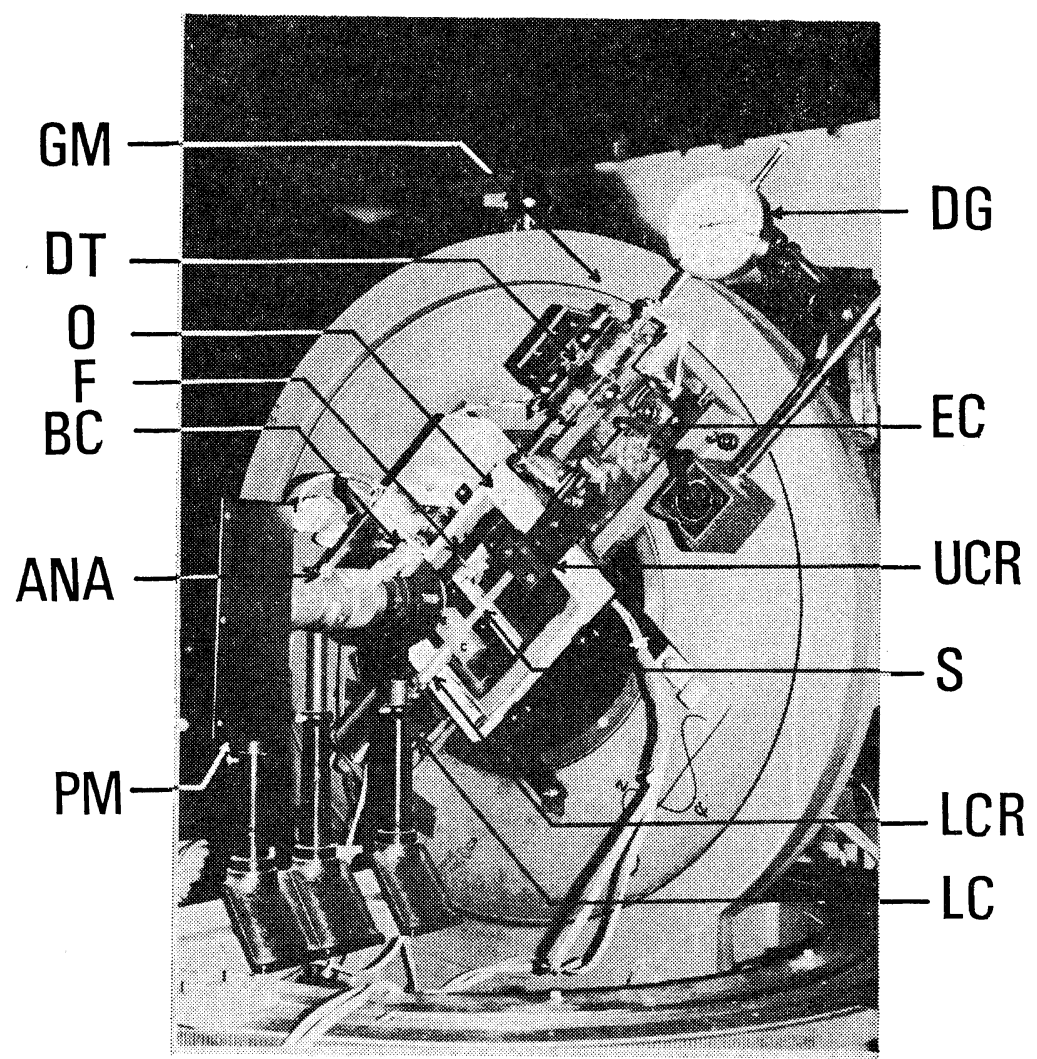

Figure 2. Front view of the dynamic birefringence apparatus illustrating the optical detecting system and the mechanical deformation device: (F) fun; (DG) dial gauge; (SB) sliding block; (UCR) upper clamp; (LCR) lower clamp; (O) oven; and (GM) goniometer. All other notations are the same as those in Figure 1. 
developed in the same laboratory, but was partially modified. A detailed description of the device will be omitted and only the modified portions will be briefly outlined. A load cell (LC) is fixed at the lower end of the clamp (LCR), as illustrated in Figures 1 and 2, and a differential transformer (DT) is attached at the upper clamp (UCR) so that both stress and strain could be simultaneously detected with the birefringence measurements. The load cell consists of a steel proof ring in which the stiffness of the ring is so arranged that its deformation during vibration is negligibly small as compared to that of the specimen. The periodic deformation of the ring during the sinusoidal deformation of the specimen (tensile stress of the specimen) is electrically transformed and amplified by a differential transformation amplifier (DA) (Figure 1); that is, the output signal is amplified by the AC amplifier, then transformed into a DC signal and again amplified by a DC amplifier. The stress signal thus converted electrically is then fed into the digital volt frequency meter (C) (TR6254/6154R, Takeda Riken Industry Co. Ltd.), in which the analogue signal is converted and displayed in digit numbers.

The sinusoidal deformation of the specimen is produced by means of an eccentric cam (EC) which is driven by a motor ( $1 \mathrm{hp}$ ); the oscillational frequency could be varied from $16 \mathrm{~Hz}$ down to $0.008 \mathrm{~Hz}$ in twelve steps with the aid of reduction gears. The eccentric cam is designed to be large enough, i.e., ca. $50 \mathrm{~mm}$ in diameter, to minimize the rotational fluctuation error of the cam; the precision of the sinusoidal displacement of the cam has been confirmed from the output signal of the differential transformer (DT) attached at the upper clamp (UCR) (tensile strain of the specimen). Moreover, the center of the eccentric cam is designed to be movable in order that the eccentricity may be continuously variable and thus the dynamic strain of the specimen may be continuously varied. The angular motion of the eccentric cam is converted to the periodic translational motion of the sliding block (SB) which is jointed with the upper clamp (UCR). The differential transformer is attached, as described above, to the sliding block so that the strain signal may be picked up from the periodic translational motion of the sliding block. Like the stress signal, the strain signal is amplified through a differential transformation amplifier (DA) and a DC amplifier, as seen in Figure 1, and displayed on the digital volt meter (C).

The gate pulse is produced by synchronization of the photoelectric switches (PS) in Figures 1 and 3 ; this method is similar in construction to that of the dynamic X-ray diffraction apparatus, ${ }^{28}$ in that a rotating disc having a narrow slit in the radial direction is placed in between the

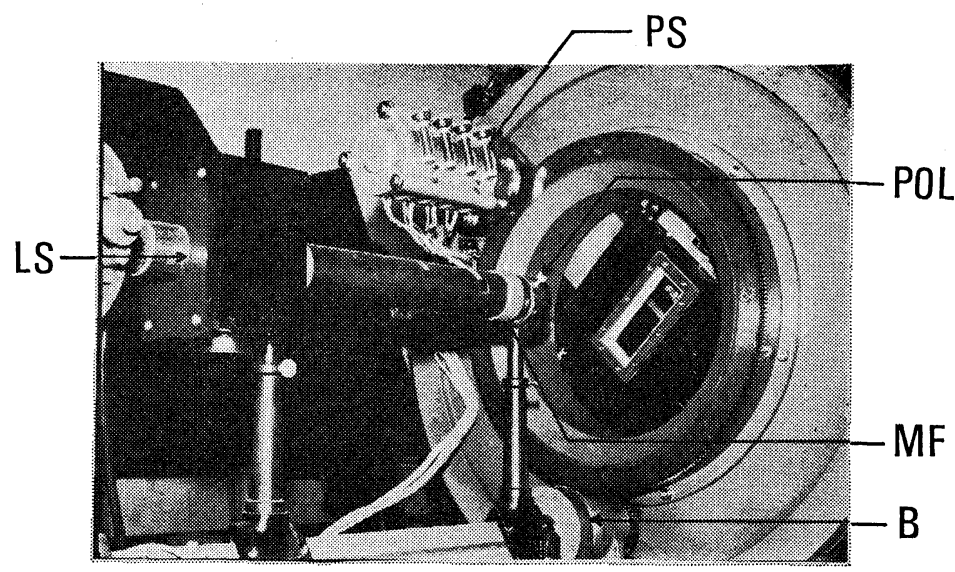

Figure 3. Back view of the dynamic birefringence apparatus illustrating the light source and a train of photoelectric switches: (B) driving belt; (PS) photoelectric switch; (LS) light source; (MF) monochromatic filter; (POL) polarizer; and (O) oven. 


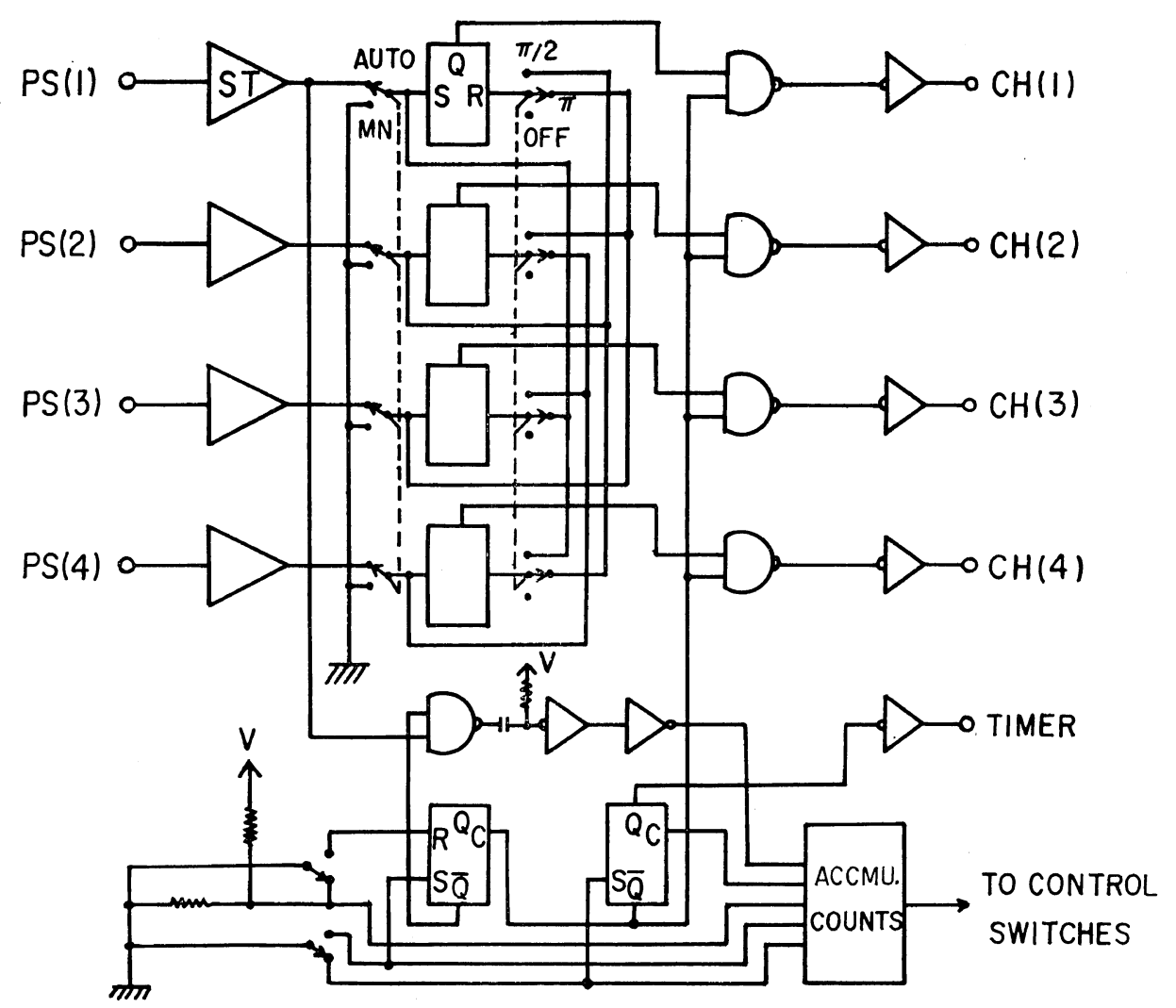

Figure 4. Schematic diagram for the gating system; the gating signal (pulse signal from photoelectric switch) is fed into the Schmitt trigger circuit (ST) which is then fed into a Flip-Flop circuit (SRQ) for the operation of "on" and "off" switching (gating) for channel $\mathrm{CH}(\mathrm{i})$ with aid of an external automatic control switches (bottom part in the figure): (S) set; (R) reset; (Q) out-put; (C) clock pulse; $(\mathrm{Q})$ inverted out-put; and $(\mathrm{MN})$ manual.

small monofilament bulb and the phototransistor, thus constituting a photoelectric switch. Four sets of photoelectric switches are set on a common shaft, and the relative position of the slit of every adjacent disc is adjusted to be shifted from its neighbor by about $\pi / 2$ radians. ${ }^{*}$ The shaft is directly connected with the eccentric cam shift. Upon rotating the disc, the narrow slit will pass through the assembly of the light bulb and phototransistor and the transistor will be activated when the light falls on it; thus, the synchronization of the photoelectric switches commences gating.

* The relative angular position of the gate pulse produced from the photoelectric switch can be adjusted more finely by moving each pair of bulb and phototransistor around the disc.
Although the synchronization of photoelectric switches is more flexible than mechanical switching, as discussed previously, ${ }^{28}$ it is still limited by the difficulty that the activation of the phototransistor by the light which passes through the narrow slit is time-dependent. Consequently, a slight frequency dependence of the photoelectric switch is unavoidable. Such a problem may no longer exist if the signal is large, but this effect cannot be ignored in the case of a small signal. Thus correction may be necessary during calibration. In view of present technology, the replacement of the photoelectric switch with an electrooptical rotary encorder (widely utilized in computor technology) may be appropriate and such a construction is now under consideration. 
The "on" and "off" gate signals are produced by feeding the pulse signal of the phototransistor, which is amplified to a certain voltage level, into a Schmitt trigger circuit (ST) (Figure 4), then feeding into a Flip-Flop circuit SRQ (S: set, R: reset, and Q: output) in order to control the gate switching for a definite phase interval, such as $\pi$ or $\pi / 2$ radians. The above gating operation for accumulating counts for a given phase interval can be automatically controlled for given cycles of oscillation by the external switches illustrated at the bottom of Figure 4. The "on" and "off" switching depends on the combination of the photoelectric switches chosen, i.e., a $\pi / 2$-sector or $\pi$-sector technique may be operable. For instance, if the $\pi$-sector technique is selected, the $\mathrm{SW}(1)$ may operate as "on" switch which may be shut off by $\mathrm{SW}(3)$ for the first phase interval, but vice versa for the third phase interval, as shown in Figure 5. Similarly, $\mathrm{SW}(2)$ and $\mathrm{SW}(4)$ will operate in the same manner for the second and fourth phase intervals.

The electric portion of the detecting system produces difficult problems in dynamic rheo-

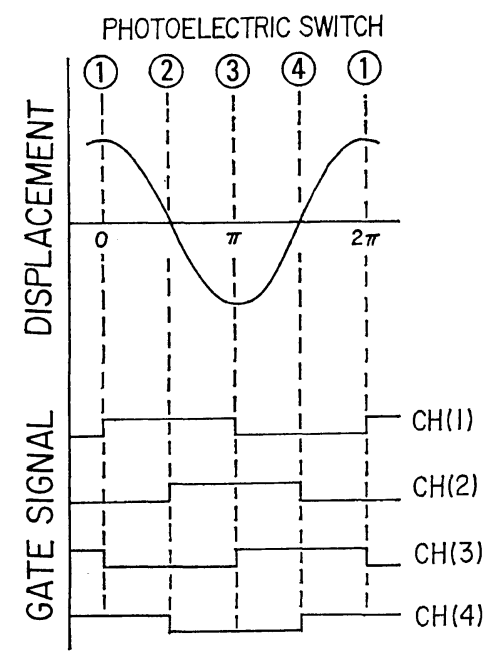

Figure 5. Schematic diagram demonstrating the $\pi$-sector technique for measuring dynamic birefringence for four particular $\pi$ radian phase intervals which are adjusted by the four sets of photoelectric switches separated from each other by $\pi / 2$ radian with respect to the sinusoidal deformation of the test specimen. optical devices since the measurements are limited by the difficulty that the amplitude of the signal or the intensity variation is very small compared to the static baseline signal; it is usually less than $10 \%$ of the static baseline in dynamic X-ray diffratcion and dynamic light scattering studies, and occasionally becomes less than $1 \%$, which may be comparable with the fluctuation of the signals themselves. In dynamic birefringence, the transmitted intensity variation is found to be about $10 \%$ of the static baseline in a low-density polyethylene, but it decreases, of course, with decreasing transparency of the specimen. In a poorly transparent specimen such as a high-density polyethylene, it is observed to be only a few percent of the static baseline. In this aspect, the measurement of dynamic birefringence by an ordinary method, i.e., the Lissajous' figure method using an oscilloscope, has difficulty achieving good precision, especially in poorly transparent specimens.

The fluctuation of the birefringent signal chiefly occurs because of the fluctuation of the incident beam, the fluctuation of high-voltage power supply, noise from the photomultiplier tube, or amplifier drift. The problem of these fluctuations may be overcome by carrying out the experiment over many cycles of oscillation. Thus the signal can be averaged by the $\pi$-sector technique for all phase intervals. The drift of the amplifier, on the other hand, cannot be eliminated by the $\pi$-sector technique. However, such a problem may no longer occur if the amplitude of the signal is made sufficiently large. The amplification of the amplitude of the signal is rather difficult since the static baseline intensity may also be amplified together with the dynamic intensity variation. Therefore, the static baseline signal is diminished by means of zero trimming of the operational amplifier (OP) before the amplification, then only the net variation portion, i.e., the amplitude of the signal, is amplified to the required gain. Accordingly, the percentage of the amplitude with respect to the static baseline has been promoted from $20 \%$ up to $90 \%$, thus enhancing the accuracy. In view of the stability of the amplifier, 40 to $50 \%$ is found appropriate, 
but in practice, 20 to $30 \%$ is sufficient enough to achieve high accuracy.

An oven which is insulated by glass fiber layers is used in this experiment. The temperature is directly controlled by four sets of heating elements of $100 \mathrm{~W}$; of these, two elements are used as a subheater for preheating and the fine temperature adjustment is controlled by the other two elements. The oven contains a fan for circulating air. The temperature can be varied from room temperature up to $180^{\circ} \mathrm{C}$. The fluctuation error of the temperature up to $90^{\circ} \mathrm{C}$ is $\pm 0.3^{\circ} \mathrm{C}$, but the fluctuation error above $90^{\circ} \mathrm{C}$ has not been checked yet. The construction of a chamber for low temperatures is still under consideration.

\section{Preliminary Results and Discussion}

Pellets of a low-density polyethylene (Sumikathene G201) of viscosity average molecular weight 42,000 and degree of branching of 2.5 $\mathrm{CH}_{3} / 100 \mathrm{C}$ are placed between aluminium foils sandwiched in between two stainless steel plates and melted in a laboratory hot press at $140^{\circ} \mathrm{C}$ for $10 \mathrm{~min}$. Then a pressure of $200 \mathrm{~kg} / \mathrm{cm}^{2}$ is applied for another $10 \mathrm{~min}$ and the system is slowly cooled down to $105^{\circ} \mathrm{C}$. The stainless steel plates are removed from the hot press and immediately plunged into an ice water bath in order to quench the film specimen thus moulded. Next the quenched film specimen is annealed at $91^{\circ} \mathrm{C}$ in a vacuum oven for two $\mathrm{hr}$ at free length state and gradually cooled down to room temperature. The thickness of the annealed specimen is $230-240 \mu$ and its density is estimated to be $0.920 \mathrm{~g} / \mathrm{cm}^{3}$ by means of the isopropynol-water density gradient tube method at $30^{\circ} \mathrm{C}$. The specimen is subjected to a light scattering study which confirms the spherulitic crystalline texture.

The specimen is kept in a dessicator for about a week before being used in the dynamic measurements in order to prevent any absorption of moisture and to allow the specimen to reach a more stable state. The film is cut into ribbons $1 \mathrm{~cm}$ wide and $8 \mathrm{~cm}$ long, which are clamped initially at a gauge length of $6 \mathrm{~cm}$, and subjected to $3.3 \%$ of static tensile stain superposed with a dynamic strain of $0.25 \%$. Before performing the dynamic measurements, the linear relationships of the differential transformer and the load cell and their analogous voltages are confirmed in order to verify the assumptions made in eq 13 through 15 . Similarly, the relation between the retardation angle of the com-

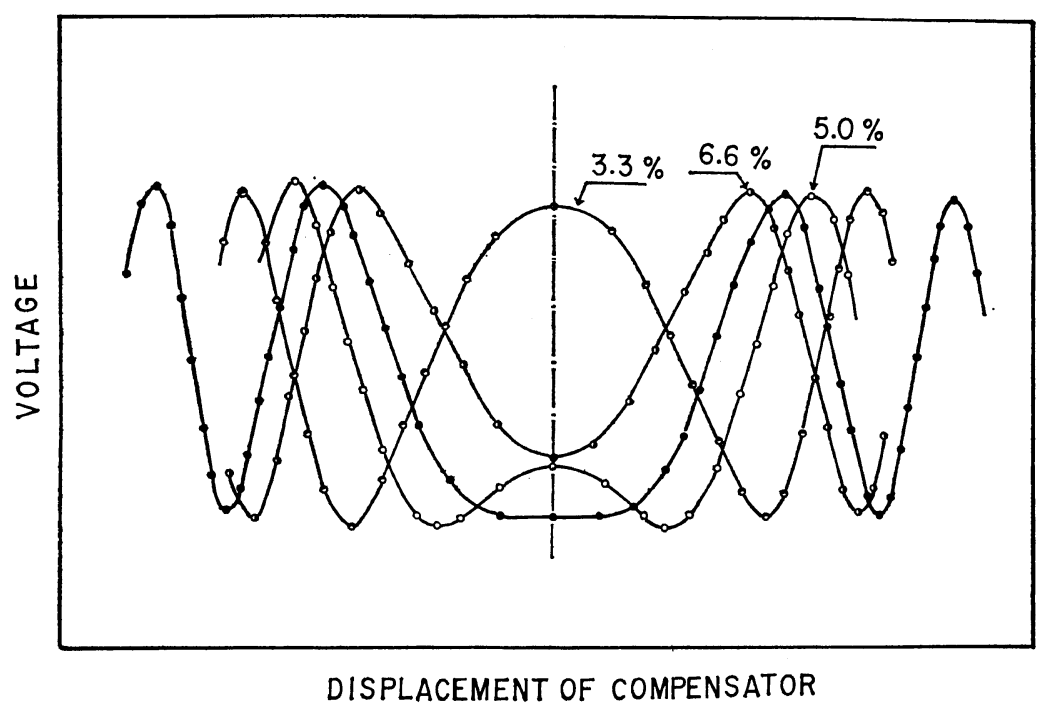

Figure 6. Relation between the displacement of compensator and the voltage generated by photomultiplier. The dots represent the curve observed without the specimen, and the other circles are those observed with the specimen of a low-density polyethylene (quenched) stretched with various \%-elongations, all at room temperature. 


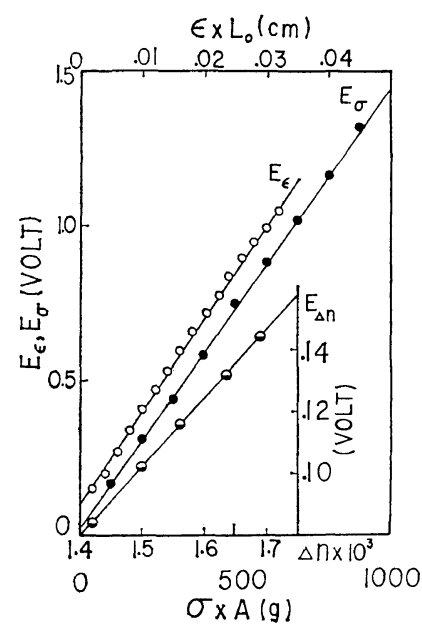

Figure 7. Relationships between the tensile stress and strain and birefringence, $\sigma, \varepsilon$, and $\Delta n$, and the analogous voltages $E_{\sigma}, E_{\varepsilon}$, and $E_{\Delta n}$. The relation of birefringence $\Delta n$ and $E_{\Delta n}$ is observed for a quenched specimen of a low-density polyethylene (G 201) at $20^{\circ} \mathrm{C}$.

pensator and the generated voltages of the photomultiplier tube is observed, as shown in Figure 6, thus fixing the position of the compensator at the center of the linear portion of the curve. The linear relation of the birefringence and the generated voltage is also con- firmed, as shown in Figure 7, together with the relation of strain and stress. It is necessary to check the frequency dependence of the amplifiers, since filters are used for the sake of signal stability, although the measurements are made in a low-frequency range. The frequency dependences of the tangent of the deviation angle $\xi$ measured with and without amplifiers are shown in Figure 8, where the dots and the open circles are optical measurements without and with operational amplifier (OP), respectively, and the open circles with perpendicular line are the mechanical measurements using the differential transformer amplifier (DA). For the optical measurements without amplifier, $\tan \xi$ does not vary with frequency, but it shows a slight frequency dependence at higher frequencies when amplifiers are used; hence corrections for the phase retardation due to the amplifiers are necessary during calibration.

All the electronic and optical devices are warmed up for about $2 \mathrm{hr}$ for the sake of stability while the specimen is heated up to the desired temperature of the experiment. A preparatory vibration is applied for about one hr at a frequency of $4.3 \mathrm{~Hz}$ to assure the vibrational steady state as well as a sort of mechanical conditioning of the specimen. Then, the

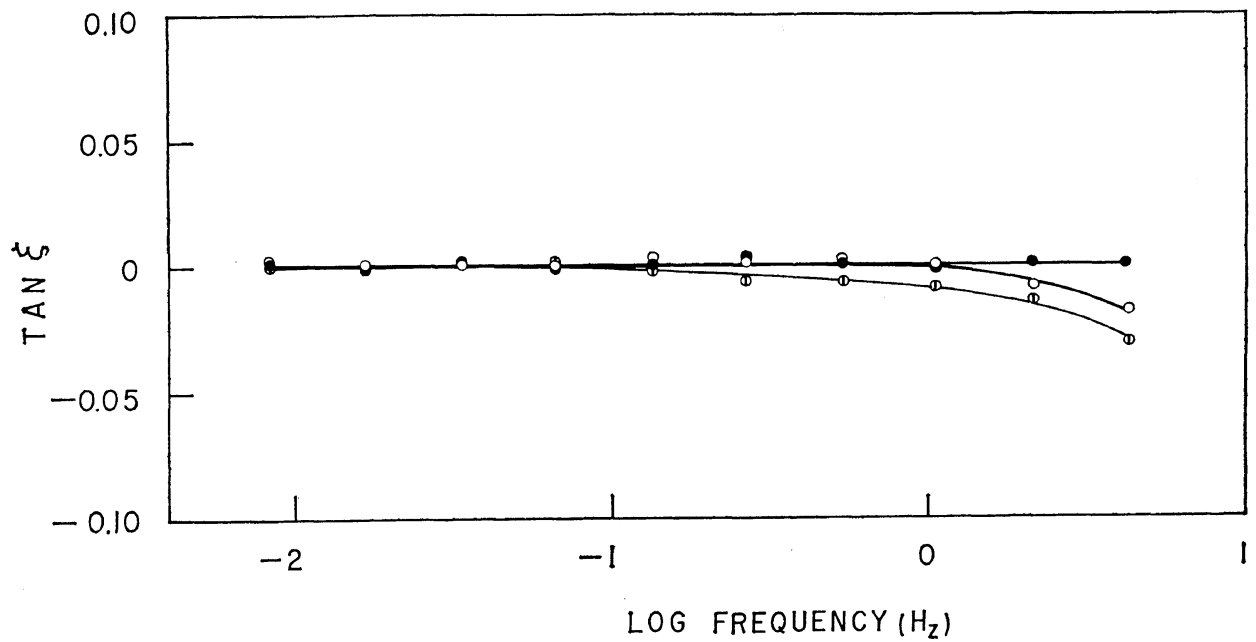

Figure 8. Determination of $\tan \xi$ and frequency dependence of amplifiers in terms of $\tan \xi$. The dots and the open circles are measured by the optical method without and with amplifier (OP), respectively, and the open circles with perpendicular line are measured by the mechanical method with differential transformer amplifier (DA). 
T. Kyu, N. Yasuda, M. Tabushi, S. Nomura, and H. Kawai

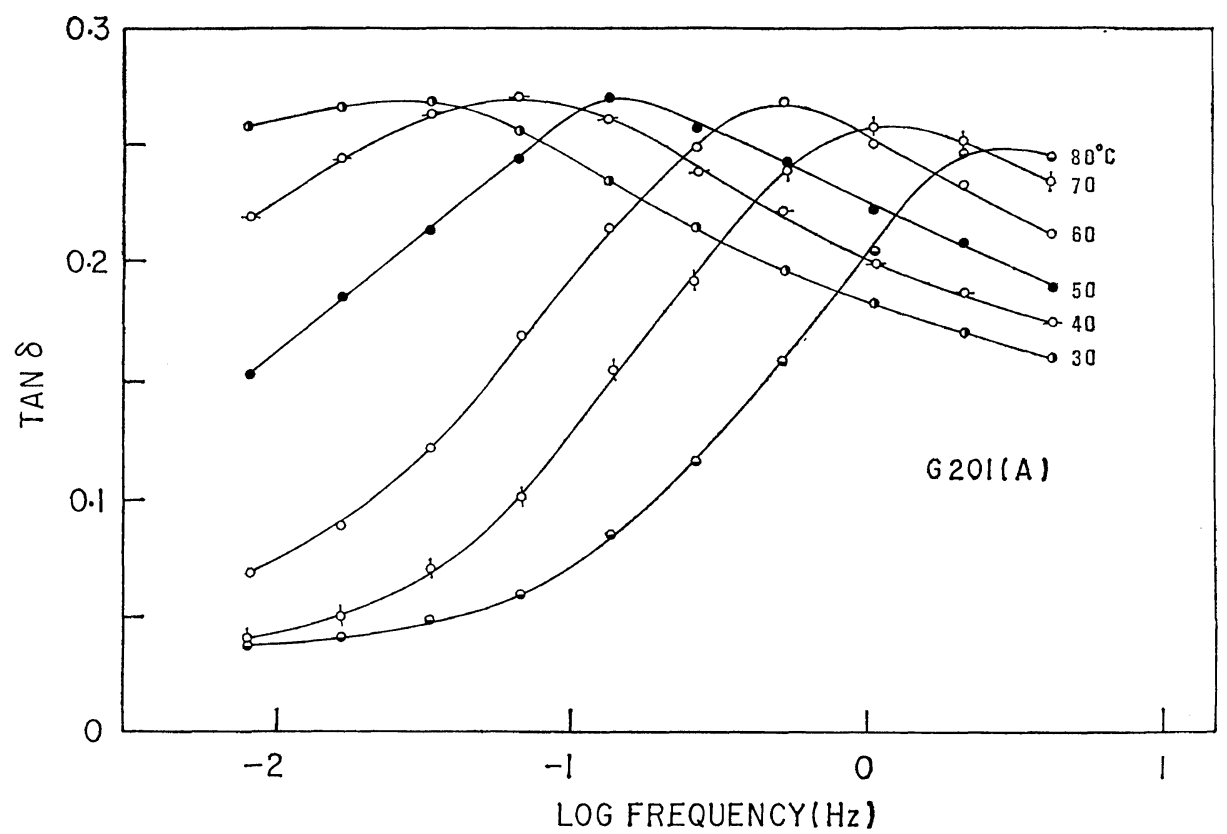

Figure 9. Frequency dependence of $\tan \delta$ for specimen of a low-density polyethylene (Sumikathene G 201, annealed) at various temperatures.

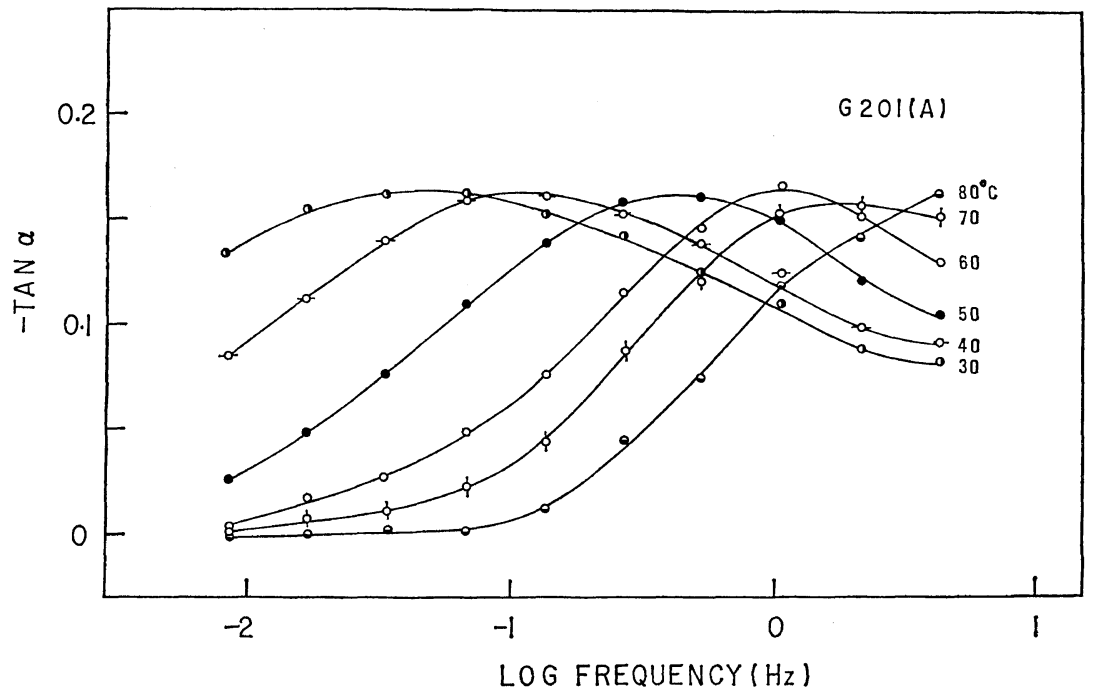

Figure 10. Frequency dependence of $\tan \alpha$ for specimen of a low-density polyethylene (Sumikathene $\mathrm{G} 201$, annealed) at various temperatures. The negative sign of $\tan \alpha$ connotes the lag in phase of the birefringence with respect to the external bulk strain.

measurements are carried out over the frequency range of $0.008-4.3 \mathrm{~Hz}$ at various temperatures from 30 to $80^{\circ} \mathrm{C}$.

Figures 9 and 10 show the frequency dependence of $\tan \delta$ and $\tan \alpha$ at various temperatures for the annealed specimen of low-density polyethylene (Sumikathen G201), where the positive sign in $\tan \delta$ indicates that the stress leads the 


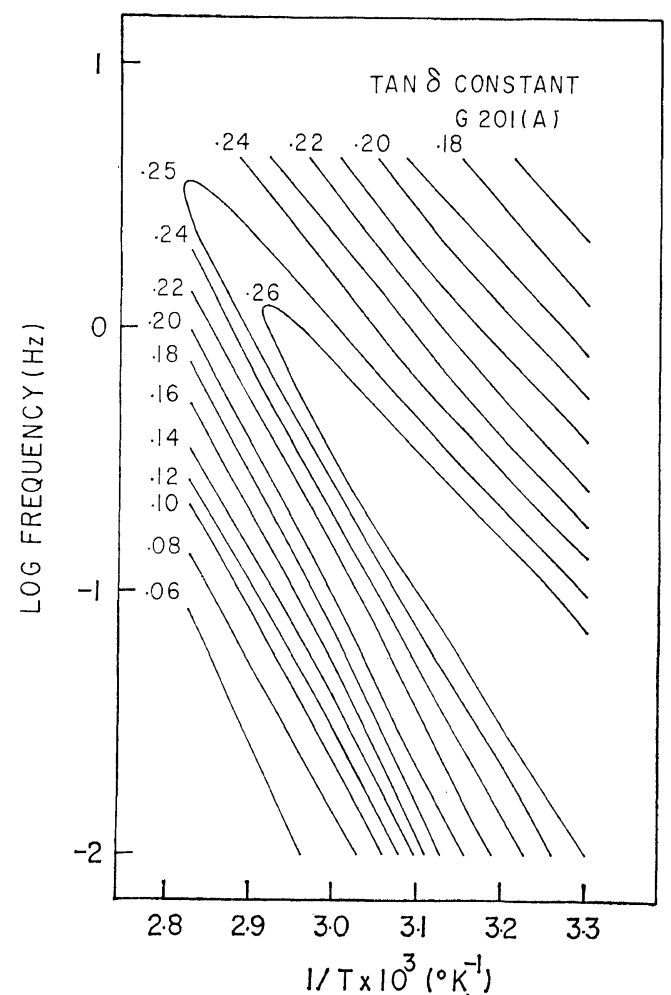

Figure 11. $\tan \delta$ contour maps for specimen of a low-density polyethylene (Sumikathene G 201, annealed). Contour lines represent the $\tan \delta$ constant lines against frequency and reciprocal of absolute temperature.

bulk strain whereas the negative $\tan \alpha$ connotes the lag in phase of birefringence with respect to the prescribed strain. In both cases, dispersion peaks are clearly observed; these shift towards the higher frequency side with increasing temperature. Although the peak position of $\tan \delta$ at a given temperature is not exactly consistent with that of $\tan \alpha$, their behaviors are very similar, connoting a good correlation between the mechanical and optical (birefringence) dispersions.

In addition, $\tan \delta$ and $\tan \alpha$ contour maps have been determined and are shown in Figures 11 and 12. The contour lines in the two maps behave in a very similar manner, running parallel to one another up to the maximum peaks. Hence, one could expect that the timetemperature superposition principle may be valid in both cases. The similarity between the two

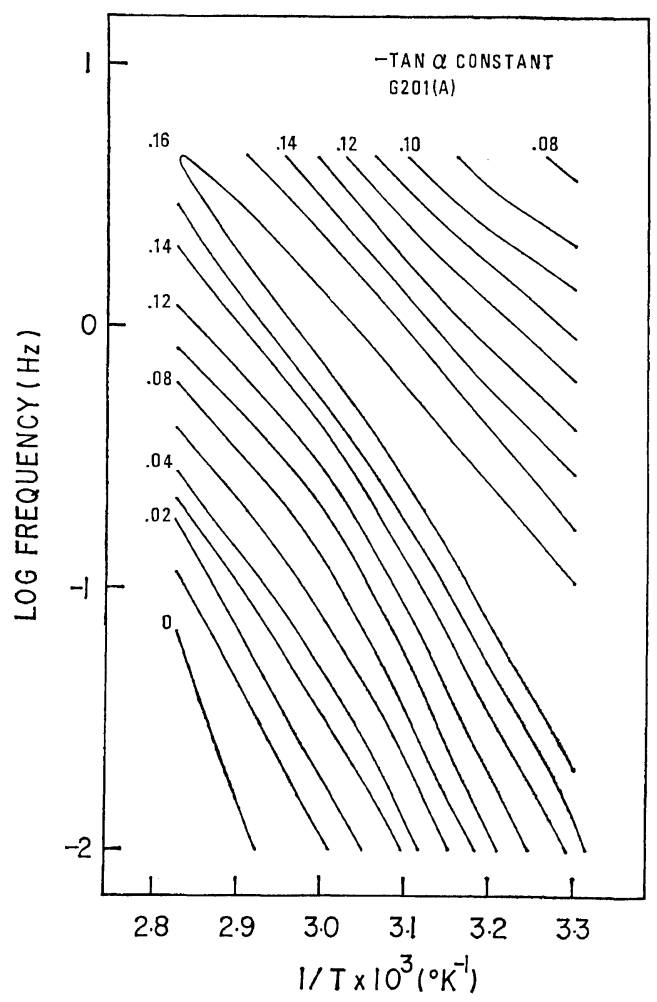

Figure 12. $\tan \alpha$ contour maps for specimen of a low-density polyethylene (Sumikathene G 201, annealed).

relaxation maps indicates the existence of a common mechanism for the two dispersions.

Figures 13 and 14 show the frequency dependence of the imaginary part of the complex dynamic modulus function $E^{\prime \prime}{ }_{T}(\omega)$ and of the real part of the complex dynamic strain optical coefficient $K_{T}^{\prime}(\omega)$, respectively. $E^{\prime \prime}$ decreases with increasing temperature and decreasing frequency; it decreases more rapidly with decreasing frequency at higher temperatures. Although $\tan \delta$ exhibits dispersion peaks in these frequency and temperature ranges, $E^{\prime \prime}$ does not show any distinct peak; it may therefore be expected that the dispersion of $E^{\prime \prime}$ is anomalously broad. On the other hand, $K^{\prime}$ increases with decreasing frequency at lower temperatures up to $50^{\circ} \mathrm{C}$, but at temperatures above $50^{\circ} \mathrm{C}$ it increases up to a certain frequency and levels off at lower frequencies. The magnitude of $K^{\prime}$ also increases with increasing temperature at higher frequencies 
T. Kyu, N. Yasuda, M. Tabushi, S. Nomura, and H. Kawai

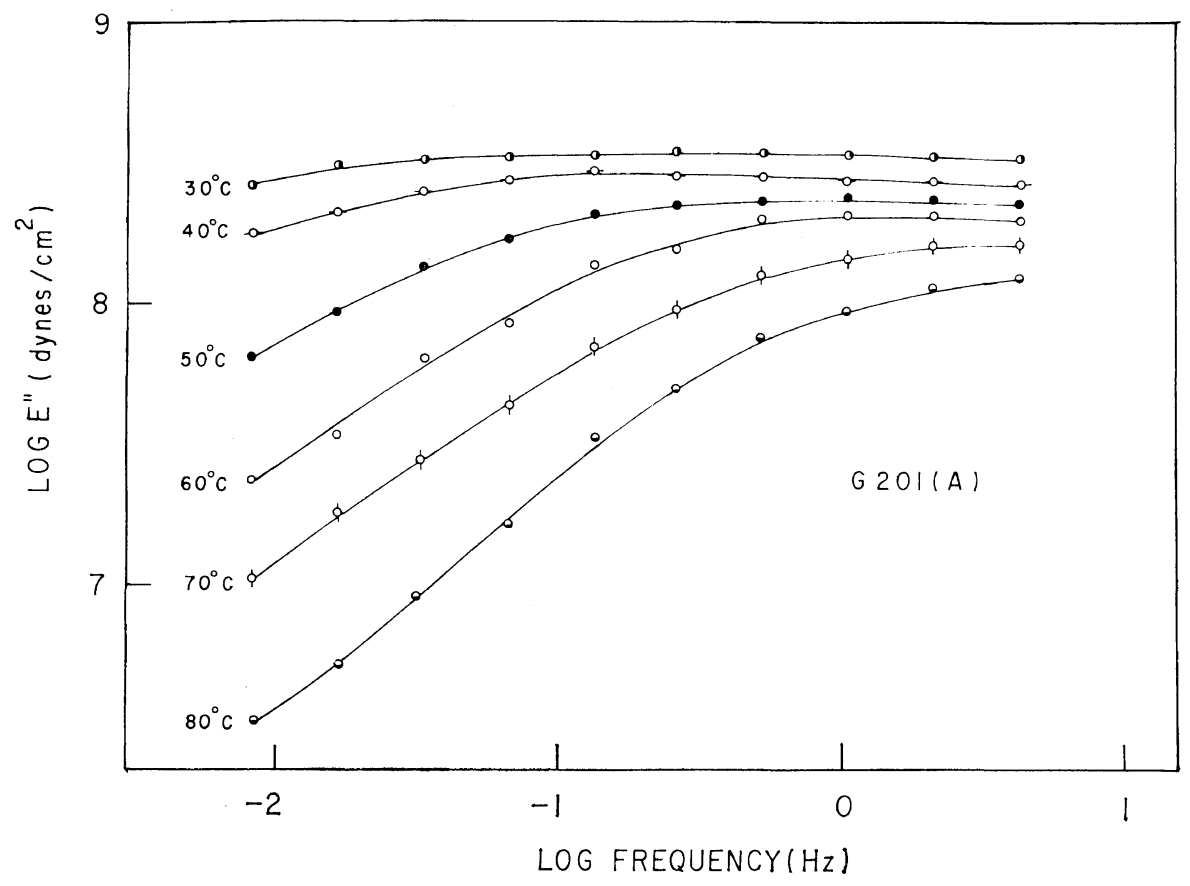

Figure 13. Frequency dependence of imaginary part of complex dynamic modulus $E^{\prime \prime}(\omega)$ for specimen of a low-density polyethylene (Sumikathene G 201, annealed) measured at various temperatures.

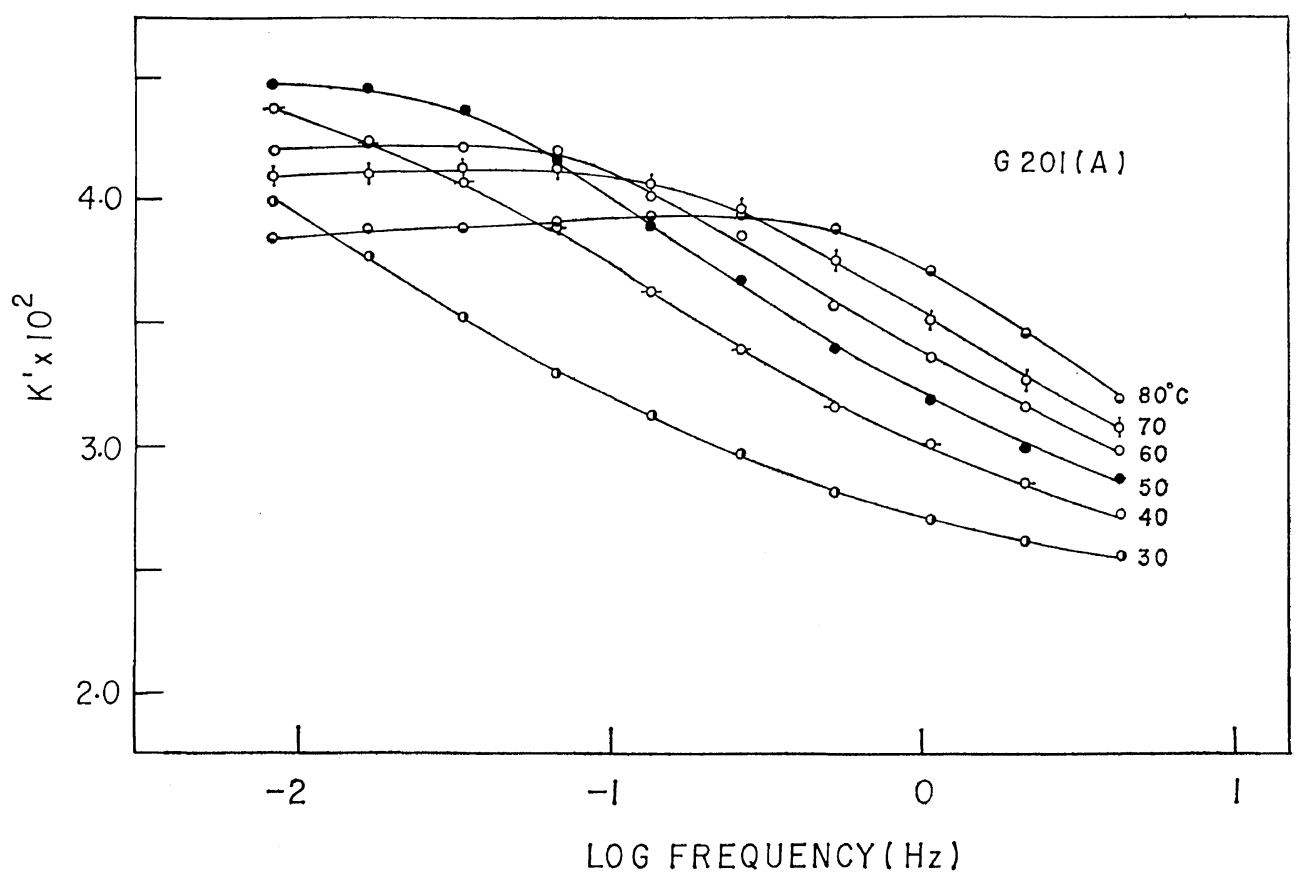

Figure 14. Frequency dependence of real part of complex dynamic strain optical coefficient $K^{\prime}(\omega)$ for specimen of a low-density polyethylene (Sumikathene G 201, annealed) measured at various temperatures. 
but decreases in contrast at lower frequencies after passing through a critical temperature. The sign of $K^{\prime}$, observed to be positive, must be explained in terms of the orientation of the crystal $c$-axis; hence the orientation of the crystal $c$-axis increases with decreasing frequency at lower temperatures but cannot further increase at higher temperatures after reaching a sort of saturated state.

The precision of $\tan \delta$ and $\tan \alpha$ are plausible, as can be noted from Figures 9 and 10. The percentage error of the loss tangents is less than $\pm 2 \%$. The precision of the real parts of the complex dynamic strain optical coefficient is extremely good, i.e., the percentage error is within $\pm 0.5 \%$. Hence, the dynamic birefringence measurements by the $\pi$-sector technique offer better precision than those by the other techniques reported earlier. ${ }^{23-25}$ The details of observed data including another kind of polyethylene will be discussed in a succeeding paper. ${ }^{30}$

Acknowledgement. The authors are indebted to the Nippon Gosei Kagaku Co. Ltd., Osaka, Japan, and the Dai-Cell Co. Ltd., Osaka, Japan, for financial support through a scientific grant.

\section{REFERENCES}

1. H. Nakayasu, H. Markovitz, and D. J. Plazek, Trans. Soc. Rheology, 5, 261 (1961).

2. N. G. McCrum, J. Polym. Sci., 60, S3 (1962).

3. N. G. McCrum and E. L. Morris, Proc. Roy. Soc., Ser. A (London), 292, 407 (1966).

4. M. Nakatani, K. Iijima, A. Suganuma, and H. Kawai, J. Macromol. Sci.-Phys., B2, 55 (1968).

5. K. Tajiri, Y. Fujii, M. Aida, and H. Kawai, ibid., B4, 1 (1970).

6. M. Takayanagi, Pure and Appl. Chem., 15, 555 (1967).

7. M. Takayanagi and T. Matsuo, J. Macromol. Sci.-Phys., B1, 407 (1967).

8. M. Takayanagi, K. Imada, and T. Kajiyama, J. Polym. Sci., Part C., 15, 263 (1966).

9. K. Okano, Rikagaku Kenkyūsho Hōkoku (Rept. Inst. Phys. Chem. Res.), 5, 273, 295 (1964).

10. Y. Wada, K. Tsuge, K. Arisawa, Y. Ohzawa, K. Shida, Y. Hotta, R. Hayakawa, and T. Nishi, J. Polym. Sci., Part C, 15, 101 (1966).

11. S. Iwayanagi and H. Nakane, Rep. Progr. Polymer Phys., Japan, 7, 179 (1964).
12. K. H. Illers, Rheo. Acta, 3, 202 (1964).

13. K. M. Sinnott, J. Appl. Phys., 37, 3385 (1966).

14. J. C. Hoffman, G. Williams, and E. Passaglia, J. Polym. Sci., Part C, 14, 173 (1966).

15. A. H. Scott, D. J. Scheiber, A. J. Curtis, J. I. Lauritzen, Jr., and J. D. Hoffman, J. Res. Nat. Bur. Stand. A, 66, 269 (1962).

16. K. Yamafuji, Kolloid-Z., 195, 111 (1964); 202, 26 (1965).

17. T. Kawaguchi, T. Ito, H. Kawai, D. Keedy, and R. S. Stein, Macromolecules, 1, 126 (1968).

18. H. Hiratsuka, M. Hashiyama, S. Tomita, and H. Kawai, J. Macromol. Sci.-Phys., B8, 101 (1973).

19. S. Onogi, D. A. Keedy, and R. S. Stein, J. Polym. Sci., 50, 153 (1961).

20. R. S. Stein, S. Onogi, and D. A. Keedy, ibid., 57, 801 (1962).

21. D. G. LeGrand and P. F. Erhardt, Trans. Soc. Rheology, 6, 301 (1962).

22. J. F. Rudd, J. Polym. Sci., Polym. Letters, 3, 345 (1965).

23. B.E. Read, Technique of Polymer Science, S.C.I., Monograph No. 17, Soc. of Chemical Industry, London, 1963, p 198.

24. R. Yamada and R. S. Stein, J. Appl. Phys., 36, 3005 (1965).

25. A. Takeuchi and R. S. Stein, J. Polym. Sci., Part A-2, 5, 1079 (1967).

26. A. Tanaka, Ph. D. Thesis, University of Massachusetts, Amherst, Mass., U.S.A., 1970.

27. T. Ito, T. Oda, H. Kawai, T. Kawaguchi, D. A. Keeky, and R. S. Stein, Rev. Sci. Instr., 39, 1847 (1968).

28. H. Kawai, T. Ito, T. Oda, H. Hiratsuka, and S. Suehiro, Mem. Fac. Eng., Kyoto University, 35, 201 (1973).

29. A. Tanaka, E. P. Chang, B. Delf, I. Kimura, and R. S. Stein, J. Polym. Sci., Polym. Phys. Ed., 11, 1891 (1973).

30. T. Kyu and M.S. Thesis, Kyoto University, Kyoto, Japan, 1974.

31. S. Onogi, T. Asada, Y. Fukui, and T. Fujisawa, J. Polym. Sci., Part A-2, 5, 1067 (1967).

32. Y. Fukui, T. Sato, M. Ushirokawa, T. Asada, and S. Onogi, ibid., Part A-2, 8, 1195 (1970).

33. S. Onogi, T. Sato, T. Asada, and Y. Fukui, ibid., Part A-2, 8, 1211 (1970).

34. Y. Fukui, Ph. D. Thesis, Kyoto University, Kyoto, Japan, 1974.

35. R. S. Stein and T. Oda, J. Polym. Sci., Part B, 9, 543 (1971).

36. T. Oda and R. S. Stein, ibid., Part A-2, 10, 635 (1972). 
T. KyU, N. Yasuda, M. TABushi, S. Nomura, and H. KawaI

37. T. Hashimoto, Ph. D. Thesis, University of Massachusetts, Amherst, Mass., U.S.A., 1970.

38. T. Hashimoto, R. E. Prud'homme, D. A. Keedy, and R.S. Stein, J. Polym. Sci., Polym. Phys.
Ed., 11, 693 (1973).

39. T. Hashimoto, R. E. Prud'homme, and R. S. Stein, ibid., Polym. Phys. Ed., 11, 709 (1973). 